\title{
Valoración de la citología exfoliativa como método diagnóstico a propósito de un Carcinoma escamoso de lengua.
}

Valuation of exfoliative cytology as a diagnostic method about a squamous Carcinoma of tongue.

Fecha de Recepción 5 de abril de 2011
Valoração da citologia esfoliativa como um método de diagnóstico de Carcinoma escamoso sobre a língua.

Aceptado para su publicación 31 de mayo de 201 I
María Silvia Almirón Jefe de Trabajos Prácticos. Cátedra de Anatomía Patológica. FOUNNE.

Roque Oscar Rosende Profesor Titular. Cátedra de Técnicas Quirúrgicas

y Anestésicas. FOUNNE.

María Eugenia Zamudio Profesora Titular. Cátedra de Biomateriales. FOUNNE.

María Alejandra Gili Profesora Adjunta. Cátedra de Histología y Embriología. FOUNNE.

\section{Resumen}

El presente trabajo tiene por finalidad comunicar el caso clínico de un Carcinoma Escamoso bien diferenciados infiltrante localizado en borde anterior de lengua el que fuera diagnosticado en una primera instancia por una citología exfoliativa y confirmado posteriormente por una biopsia. En la cavidad bucal suele utilizarse con mas frecuencia la biopsia que la citología como método diagnóstico, sin embargo estas técnicas no deben competir entre ellas sino mas bien complementarse. En comparación con la biopsia, la toma de muestra citológica es mas sencilla, indolora y menos invasiva. El examen citológico puede revelar la presencia de células cancerosas en etapas tempranas pero este se encuentra sujeto en la mayoría de los casos al resultado final de la biopsia. La citología es un adecuado método auxiliar en el diagnóstico precoz de lesiones sugerentes de malignidad pero siempre en combinación con la biopsia. El $50 \%$ de los tumores de la boca se localizan en la lengua. La edad promedio de aparición es a los 60 años, con una relación hombre/ mujer de 3/ I. El $95 \%$ son carcinomas de células escamosas, y el resto está constituido por el carcinoma verrugoso y por los tumores de las glándulas salivales menores. La etiología se asocia fuertemente con el abuso de alcohol y tabaco al igual que en otros carcinomas epidermoides de cabeza y cuello. También se demostró que las úlceras traumáticas de lengua pueden malignizarse, ante la presencia de factores irritativos crónicos de larga data. En el presente trabajo presentamos un caso de carcinoma escamoso bien 
diferenciado, infiltrante de mucosa oral, diagnosticado con una citología exfoliativa y confirmado por una biopsia.

\section{Palabras Claves}

Tumormalignoepitelial-CarcinomaEpidermoideo - Lengua - Citología exfoliativa - Biopsia.

\section{Abstract}

The present work has for purpose communicate the clinical differentiated well case of a Scaly unfiltering Carcinoma located in previous edge of language the one that should diagnose in the first instance by a cytology exfoliativa and confirmed later by a biopsy.

In the mouth cavity frequency is in the habit of using with mas the biopsy that I diagnose the cytology like method, nevertheless these technologies must not compete between them but rather to complement itself, In comparison with the biopsy, the capture of cytological sample is mas simple, painless and less invasive. The cytological examination can reveal the presence of cancerous cells in early stages but this one is fastened in the majority of the occasions to the final result of the biopsy, that is to say the cytology suitable auxiliary method is in the precocious diagnosis of suggestive injuries of malice but always in combination with the biopsy.

$50 \%$ of the tumors of the mouth is located in the language. The average age is 60 years, with a relation hombre/wife of $3 / 1.95 \%$ is carcinomas of scaly cells, and the rest is constituted by the warty carcinoma and by the tumors of the salivary minor glands. The etiology associates strongly with the abuse of alcohol and tobacco as in other carcinomas epidermoides of head and neck. Also there was demonstrated that the traumatic sores of language can malignizarse, before the presence of factors irritativos chronic of long byline In the present work let's sense beforehand a case of carcinoma epidermoide differentiated good, unfiltering of mucous oral, diagnosed with a cytology exfoliativa and corroborated by a biopsy.

\section{Keywords}

Malignant tumor epitelial - Carcinoma Epidermoideo - Language - cytology exfoliativabiopsy.

\section{Resumo}

Este trabalho visa relatar um caso de carcinoma de células escamosas bem diferenciado infiltrativo localizado na borda anterior da língua, que foi diagnosticada em primeira instância pela citologia esfoliativa e mais tarde confirmado pela biópsia da cavidade oral é geralmente usado com mais freqüência a citologia da biópsia como método diagnóstico, porém estas técnicas não devem competir, mas sim complementá-los, em comparação com a biópsia, a amostragem citológica é mais simples, indolor e menos invasivo. A citologia pode revelar a presença de células cancerosas em estágios iniciais, mas isso é assunto na maioria dos casos o resultado final da citologia de biópsia é um método auxiliar adequado para o diagnóstico precoce de lesões sugestivas de malignidade, mas sempre em combinação com biópsia, 50\% dos tumores da boca estão localizados na língua. A idade média é de 60 anos, com uma relação masculino/feminino de $3 / 1$. $95 \%$ são carcinomas espinocelulares, eo resto são o carcinoma verrucoso e tumores das glândulas salivares crianças. A etiologia é fortemente associada com o abuso de álcool e rapé como em outros carcinomas de células escamosas da cabeça e pescoço. Também demonstrou que as úlceras traumáticas podem se tornar a língua maligna, na presença de fatores irritativos crônicos Este trabalho apresenta um caso de longa data do carcinoma de células escamosas bem diferenciado, infiltrando-se na mucosa bucal, diagnosticado com um exame de Papanicolaou e confirmado pela biópsia.

\section{Palavras chave \\ Carcinoma epitelialmalignidade - Epidermoideo- Language-citologia esfoliativa- biópsia.}

\section{Introducción}

El carcinoma de células escamosas de cabeza y cuello es un tumor epitelial maligno muy agresivo que constituye la sexta neoplasia más común en nuestros días'. Aproximadamente el $50 \%$ se localizan en la lengua ${ }^{2}$.

Generalmente este tumor afecta a individuos de mediana edad o ancianos que han sido bebedores y/o fumadores ${ }^{3,4}$. Generalmente la incidencia es mayor en hombres que en mujeres En cuanto al sexo y la edad, en términos generales, la inciden- 
cia del COCE (carcinoma oral de células escamosas) se incrementa con la edad habitualmente mayores de 45 años en un $90 \%$, aunque la edad media en la que se presenta varía en función de su localización. Para la mayoría de los autores la etapa de mayor incidencia estaría comprendida entre los 45 y 60 años; sin embargo otros informes alargan la edad media de aparición a los 64 años ${ }^{5}$.

En un principio se habla de una importancia y predominancia similar del hábito de fumar tabaco y el consumo de alcohol como factores etiológicos del cáncer de la cavidad oral y concretamente del cáncer de lengua. Sin olvidar la existencia de otros factores etiológicos revisados en la literatura como el estado de la dentición ${ }^{7}$, los hábitos de higiene oral ${ }^{8}$, la nutrición y las infecciones virales Shiboski $\mathrm{CH}$. Et al. también hace hincapié en la posible relación que puede existir entre el riesgo de padecer cáncer de lengua y la infección por Virus del papiloma humano (VPH), ya que la incidencia de ambas enfermedades aumenta de manera paralela, estableciendo finalmente una relación entre el riesgo de COCE, historial sexual y evidencia de infección por $\mathrm{VPH}^{8}$.

La citología exfoliativa oral se define como el estudio e interpretación de los caracteres de las células que se descaman, natural o artificialmente, de la mucosa oral. Consiste en observar al microscopio óptico la morfología de las células epiteliales superficiales después de su toma, fijación y tinción?. Es una técnica sencilla, no agresiva, relativamente indolora y bien aceptada por los pacientes, por lo que podría ser útil en el diagnóstico precoz del cáncer oral. Sin embargo, el uso de la citología exfoliativa oral para el diagnóstico de atipías epiteliales y especialmente del carcinoma oral de células escamosas ha perdido importancia, sobre todo debido a su baja sensibilidad representada por el elevado número de resultados falsos negativos. Se atribuye esta baja sensibilidad a diversos factores: toma inadecuada de la muestra, error en la técnica e interpretación subjetiva de los hallazgos citológicos ${ }^{10}$. En relación a la toma de la muestra el uso del cytobrush parece aumentar el número de células recogidas por muestra y permite una mejor distribución de las mismas en el portaobjetos, lo que podría aumentar la sensibilidad de la técnica" ${ }^{\prime \text {. En los }}$ últimos tiempos, el desarrollo del análisis cuantitativo, la citomorfología, el análisis del ADN, la detección de marcadores tumorales y los métodos de diagnóstico molecular han contribuido al resurgir de esta técnica ${ }^{12}$.

El cáncer oral involucra una serie de pasos, cada uno caracterizado por una acumulación secuencial de defectos genéticos seguidos de una proliferación por clonación. Los procesos neoplásicos se inician en un epitelio normal, lo modifican a un epitelio hiperplásico, displásico, carcinoma in situ y finalmente en un carcinoma invasivo ${ }^{13}$.

Los tejidos blandos de la boca manifiestan una variedad de patologías sistémicas con compromiso cutáneo, entre ellas se encuentran lesiones como líquenes orales planos, leucoplasias, candidiasis, queratosis, papilomas, úlceras traumáticas, infecciones por HPV, entre otros ${ }^{14}$. Las leucoplasias y líquenes, con y sin infección con HPV, constituyen lesiones frecuentes de la mucosa bucal con características premalignas o precancerosas. Las leucoplasias pueden transformarse en carcinomas en un $\mathrm{I}-10 \%$ y los líquenes en un $5 \%$, dependiendo del tipo y del grado de displasia epitelial observado ${ }^{15}$.

La transformación maligna en la cavidad oral depende en cierto modo del sitio anatómico que se ve afectado (labios, lengua, piso de la boca, paladar, gíngiva, mucosa bucal y orofaringe), de los numerosos factores endógenos y exógenos que afectan el crecimiento de las células malignas en cada uno de estos sitios y de la relación con el suministro de nutrientes ${ }^{16,17}$.

La citología exfoliativa de la mucosa oral muestra alteraciones morfológicas relevantes que coinciden con patrones de carcinogénesis mencionados en la literatura. La forma y el cambio en el tamaño del núcleo, como así también el aspecto sucio del preparados son indicadores de cambios relacionados con la carcinogénesis ${ }^{14,18}$. Conjuntamente con estas características, en la tinción con PAP se puede observar una coloración naranja, característica de hiperqueratinización ${ }^{18}$.

En nuestro país, la citología exfoliativa de la mucosa oral, no se utiliza sistemáticamente como método de prevención o seguimiento de condiciones patológicas orales, sin embargo estudios llevados a cabo por autores como Silverman et al $^{18,19}$ en poblaciones de la India, identificaron precisamente cánceres orales en las CE (citología exfoliativa) en el $90 \%$ de los casos, la que fue evidenciada cuando éstas resultaron sospechosas, mientras que las biopsias en los mismos 
pacientes resultaron negativas en un comienzo y luego confirmaron diagnóstico de cáncer ${ }^{19}$.

En los últimos años se ha utilizado un procedimiento diagnóstico de lesiones orales malignas, llamado "brush biopsy", en el que se realiza la toma con un cepillo de diseño particular que permite penetrar en el espesor de la mucosa y recoger material representativo de las lesiones $^{20,21}$. Este método ha sido diseñado para que extraiga células desde la capa superficial a la basal del epitelio, $y$ de ese modo permita la detección de aquellas que son anómalas. La valoración de "malignidad" o "benignidad" se realiza mediante un análisis asistido por ordenador.Como los mismos defensores de este procedimiento señalan, esta técnica no sustituye en ningún caso a la biopsia clásica, y su uso es complementario.

\section{Presentación del Caso}

Concurre Al Servicio de Odontología del Centro de Atención primaria de la salud "Construir Salud" una paciente de sexo femenino de 50 años de edad por la consulta de una lesión ubicada en el borde lateral anterior de la lengua. Se realizó la historia clínica médica odontológica correspondiente donde la paciente relata que la lesión tiene una evolución de aproximadamente cuatro años. Se presentaba como una lesión clínica de afta y por la que había recibido tratamiento específico para esta afección. Al interrogatorio la paciente relata que no fuma $y$ que no consume alcohol. Refiere dolor $y$ ardor exacerbado principalmente a lo caliente con odinofagia y dificultad para hablar.

Destaca que se encuentra bajo tratamiento ginecológico por presentar una displasia moderada de cuello uterino (SIN II) con presencia de HPV. En la última consulta realizada después de este período a la inspección intra oral se constato un cambio clínico en la lesión, donde se observó una masa tumoral vegetante, elevada exofística y en parte ulcerada de un tamaño aproximado, de $15 \mathrm{~mm}$.en sentido mesio distal por $10 \mathrm{~mm}$, en sentido vestíbulo lingual de límites definidos, con áreas de coloración rojiza, consistencia sólida e indurada en uno de sus bordes.

Se observó, sepsis bucal, pérdida de piezas dentarias, caries, enfermedad gingival y periodontal y en relación con el tumor la presencia de un premolar inferior con bordes filosos. A la palpación de la cadena ganglionar cervical no se detectaron adenopatías.

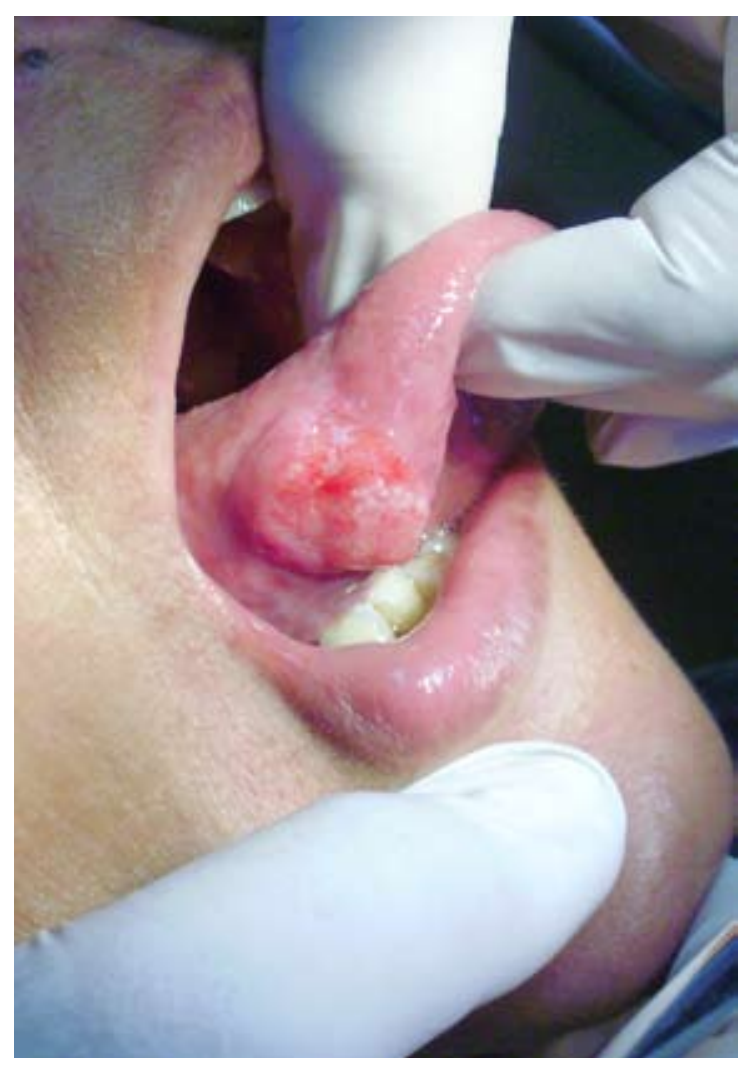

Foto I. Visión clínica de la lesión.

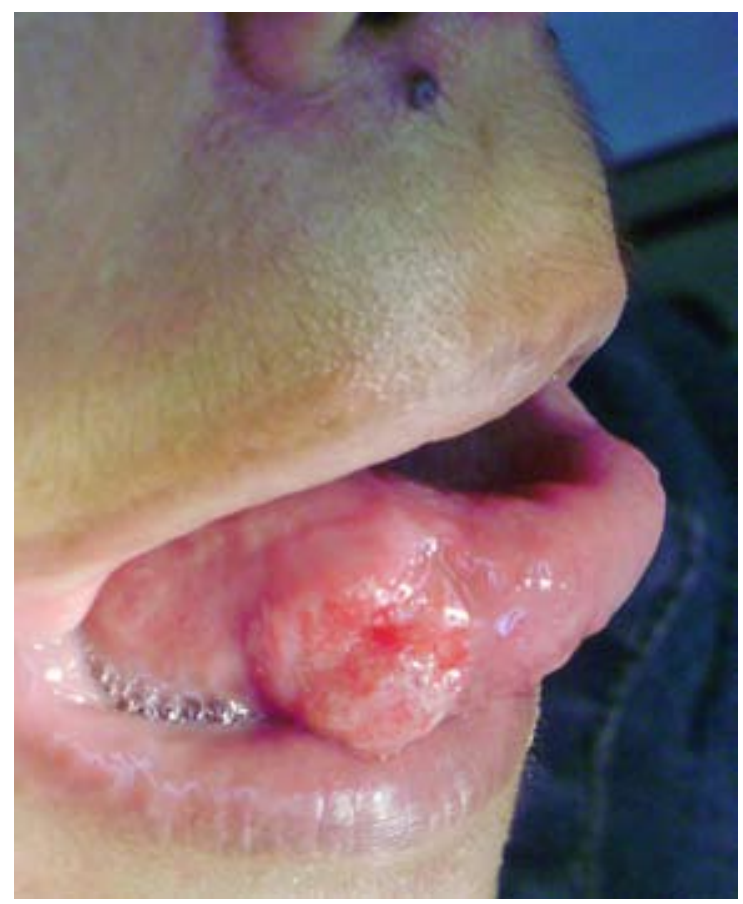

Foto 2. Visión clínica de la lesión. 
Se explicó a la paciente los pasos a seguir y con el debido consentimiento informado se procedió a realizar en primer lugar el pulido de la pieza dentaria en relación con la lesión, inmediatamente se le realizó una citología exfoliativa con cytobrush en dicha zona y se fijó con spray de cabello. El material fue remitido al Servicio de Anatomía Patológica para su correspondiente procesamiento utilizándose la técnica de coloración de Papanicolau modificada.

Los extendidos citológicos muestran células pavimentosas atípicas con anomalías citológicas inflamatorias, macrocariosis, anisonucleosis dispersas, abundantes detritus, bacilos, cocos y aisladas hifas micóticas, leucocitos polimornucleares todo sobre un fondo sucio.

El informe citológico es: extendido trófico LIE (lesión intraepitelial escamosa) de bajo grado. Inflamación crónica reagudizada.

En una cita posterior se le informa a la paciente que es necesario realizar una biopsia para confirmar el diagnóstico citológico.

Se realizó la biopsia incisional tomando dos partes de tejido representativo incluyendo bordes de la lesión y tejido circundante.

Se fijó en formol al 10\% y se envió al Servicio de Anatomía Patológica dela Facultad de Odontología de la Universidad Nacional del Nordeste. Se realizó la técnica de rutina con inclusión en parafina y coloración H/E. Los preparados histopatológicos mostraban dos fragmentos de mucosa con ulceración cubierta por exudado fibrinoleucocitario con proliferación epitelial atípica de estirpe pavimentosa con remolinos córneos macro y anisocariosis, dispuesto en nidos sólidos y cordones en el corion subyacente. Coexiste intenso infiltrado inflamatorio mono y polimorfonuclear. Márgenes de resección comprometidos por la lesión. El Informe histopatológico definitivo es de Carcinoma epidermoide Bien Diferenciado, Ulcerado e Infiltrante. (BRODERS I). La paciente fue derivada al Servicio de Oncología de la Obra Social" Construir Salud" par efectuar el tratamiento pertinente.

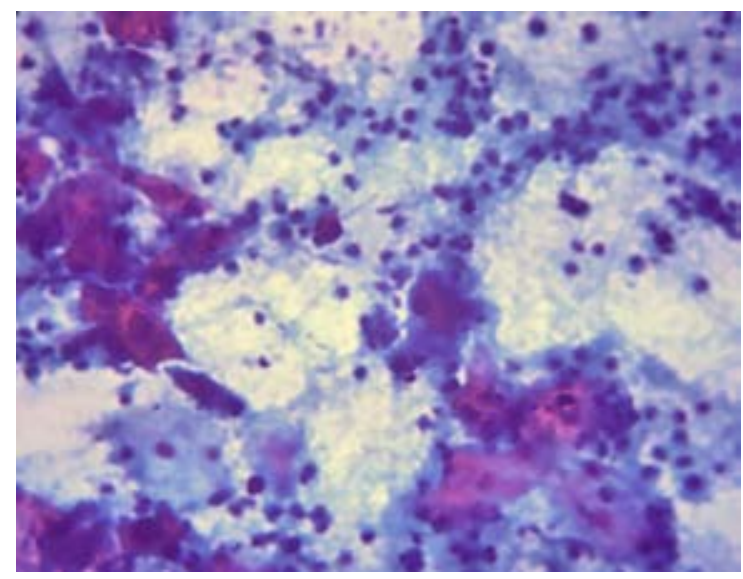

Foto 3. Citología Exfoliativa: Células Pavimentosas atípicas con abundante infiltrado inflamatorio mono y polimorfonuclear.40X.Técnica PAP.

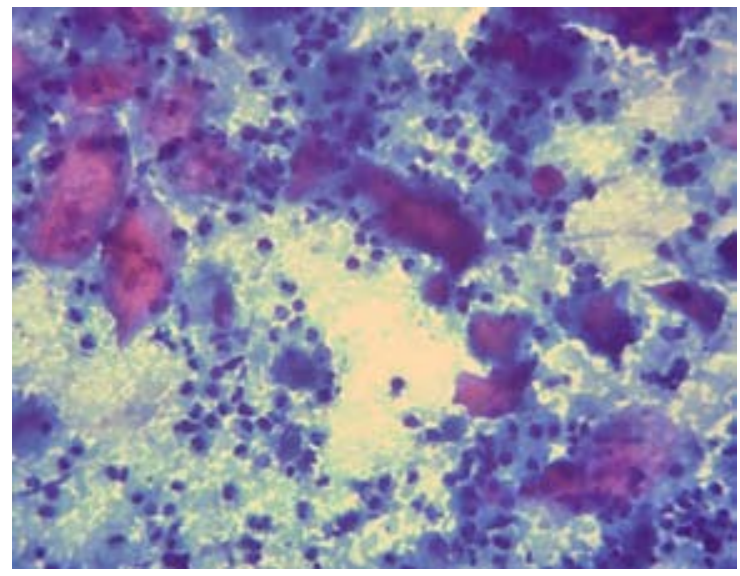

Foto 4. Citología Exfoliativa: Células epiteliales atípicas con pérdida d la relación núcleo citoplasma, marcada hipercromasia macrocariosis y anisonucleosis 40X. Técnica PAP.

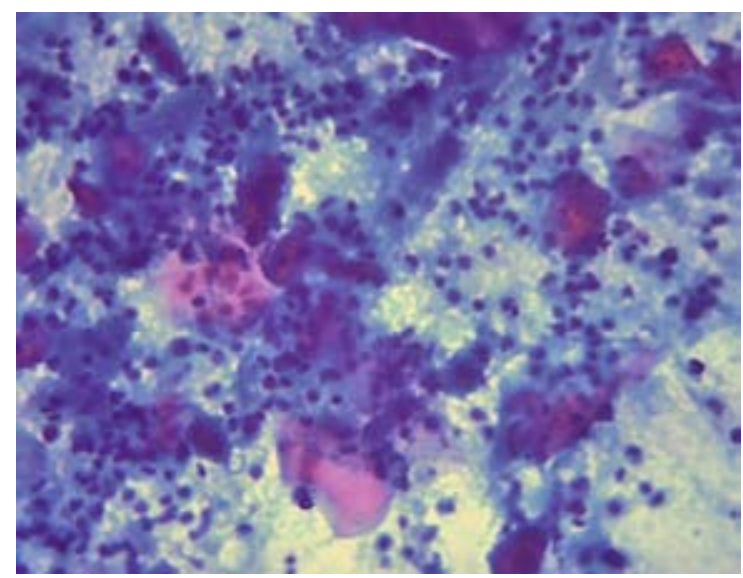

Foto 5. Citología Exfoliativa: Fondo sucio con detritus y abundantes bacilos y cocos entre las células epiteliales 40X Técnica PAP. 


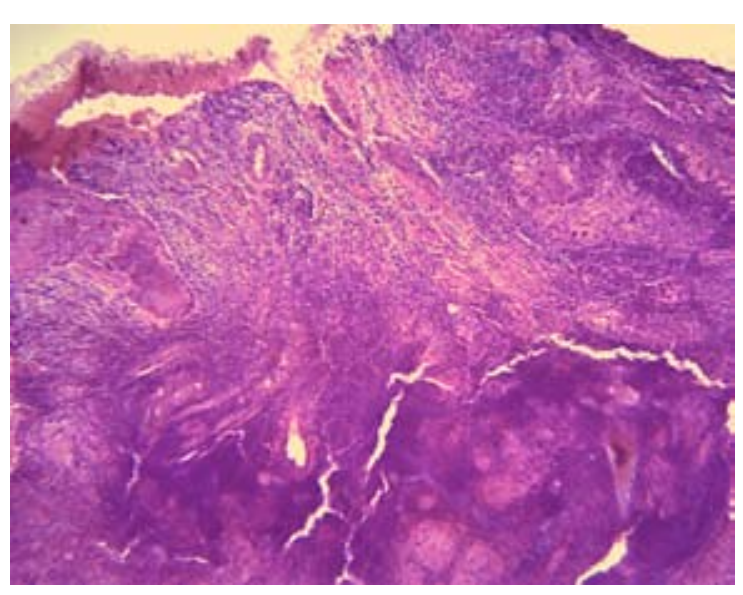

Foto 6. Mucosa lingual con Ulceración y exudado fibrinoleucocitario. 10 X. H/E.

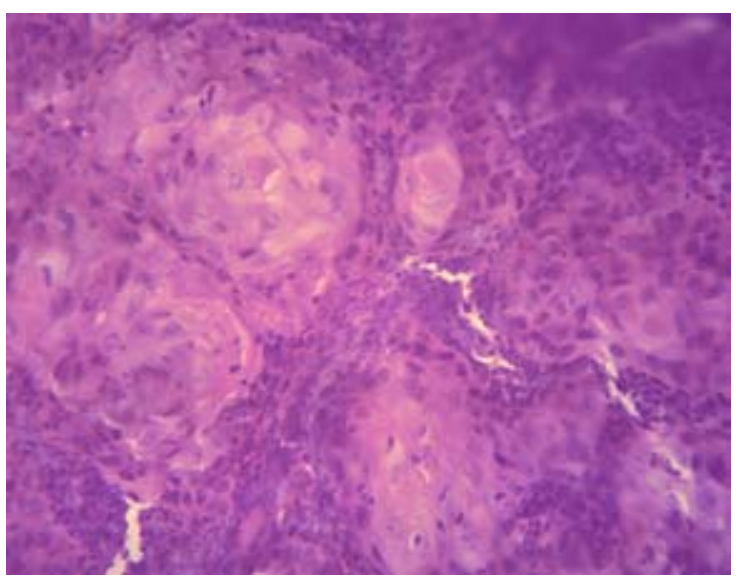

Foto 7. Intenso infiltrado mono y polimorfonuclear entre células epiteliales atípicas 40 X. H/E.

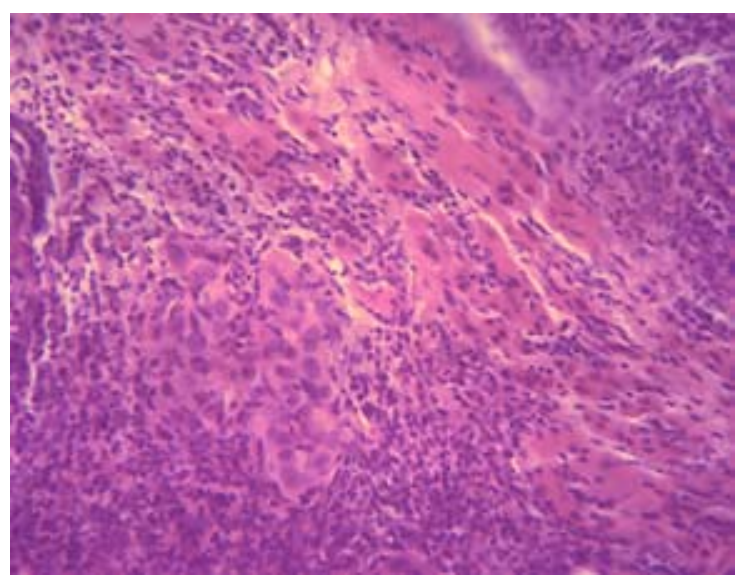

Foto 8. Remolinos córneos y acúmulos de células atípicas con macro y anisocariosis 40 X. HIE.

\section{Discusión}

El carcinoma epidermoide originado en mucosas de cabeza y cuello ha mostrado en la última década un incremento en su frecuencia.

Actualmente el cáncer originado en cavidad oral, principalmente en la lengua, ocupa el sexto lugar mundial de todas las neoplasias 22 .

El comportamiento epidemiológico de esta neoplasia tiende a cambiar desde los últimos 10 años; actualmente la frecuencia de esta neoplasia en jóvenes (menores de 45 años), sobre todo mujeres y no consumidores de alcohol ni tabaco ha aumentado en forma importante ${ }^{23}$.

Todas las neoplasias epiteliales de la cavidad bucal son precedidas por un carcinoma in situ que en 2/3 de los casos carece de signos clínicos característicos ${ }^{24,25}$; sin embargo estos, pueden ser evidenciados en la citología exfoliativa, técnica que cuando es realizada por individuos con experiencia, bien entrenados, ofrece un grado extremadamente alto de confiabilidad. Bajo estas circunstancias, un diagnóstico positivo de malignidad debe alertar al profesional ${ }^{25}$.

La literatura médica destaca que la citología exfoliativa puede ser un importante auxiliar en los carcinomas bucales incipientes que tienen superficies ulceradas, mientras que las lesiones secas, costrosas, muy queratósicas o necrosadas, producen muestras malas y no se prestan para el examen citológico ${ }^{26}$. Las principales ventajas clínicas de la citología exfoliativa se deben a que es una técnica rápida, no agresiva y relativamente indolora, muy bien aceptada por los pacientes, lo que permite que sea practicada repetidamente en programas preventivos de screening, en lesiones sospechosas ya identificadas o en el seguimiento de lesiones malignas postratamiento.

Los métodos diagnósticos clásicos para las lesiones cancerosas y precancerosas orales son el examen clínico y el estudio histopatológico del material obtenido por biopsia. El análisis biópsico es todavía la técnica más aceptada para determinar de un modo fiable la naturaleza de las lesiones de la mucosa oral. Además, el estudio biópsico con ser fundamental, no deja de ser un método diagnóstico con sensibilidad limitada, en el que prima en gran medida la subjetividad del patólogo observador. Todos estos aspectos nos indican la importancia que tiene el descubrir y desarrollar nuevos métodos diagnósticos, así como mejorar los ya conoci- 
dos, y buscar también dianas terapéuticas para la enfermedad neoplásica oral ${ }^{21,27}$. Es importante que las metodologías sean sencillas, poco cruentas y fiables, y que nos permitan realizar un diagnóstico y seguimiento satisfactorios en los pacientes con lesiones precancerosas y cancerosas.

\section{Conclusiòn}

Concluimos que la mayoría de las citologías exfoliativas en lesiones bucales tiene un alto porcentaje de sensibilidad, alto valor predictivo, una coincidencia diagnóstica elevada y un gran porcentaje de eficiencia global, lo que demuestra su importancia como método no invasivo de diagnóstico.

La utilización de la citología exfoliativa puede llegar a obtener muestras para la aplicación de técnicas sofisticadas de diagnóstico, citomorfometría, análisis del contenido de ADN, y análisis molecular, actualmente estos estudios han ganado espacio como métodos de diagnóstico fiable del cáncer oral en sus estadios más precoces.

Es importante señalar que la citología nunca debe prevalecer sobre el estudio biópsico clásico y que ante toda lesión clínicamente sospechosa, aún con el diagnóstico citológico de benignidad es mandatario realizar una biopsia.

Es así que el odontólogo debe estar comprometido y debidamente entrenado para la detección del estado inicial de la enfermedad por su carácter asintomático y por tornarse confuso el diagnóstico diferencial dada la variedad de manifestaciones bucales de enfermedades sistémicas semejantes. No obstante, es importante también encontrarse entrenado en la realización de exámenes rutinarios en pacientes sin lesiones orales pero con la presencia de factores de riesgo, como el consumo de alcohol y tabaco y el carácter multifactorial que presenta.

Debe entenderse que la capacidad de control del cáncer en la cavidad bucal depende básicamente de dos aspectos fundamentales, la prevención, el control en la interrupción de los factores de riesgo y el diagnóstico precoz. Ello implica la necesidad de generar campañas educativas, locales, regionales y nacionales con agrupaciones académicas y científicas contando con la intervención y activa participación de la Salud Pública.

\section{Bibliografía}

I. Vokes EE., Weichsalbaum RR., Lippman SM. Head and neck cancer. N Engl J Med 1993; 328: 184 - 94.

2. Boring $C$ C, Squires TS, Tong T. Cancer statistics. CA Cancer J Clin 1991; 41: 19 - 36.

3. Blot WJ, McLaughlin JK, Winn DM, Austin DF, Greenberg RS. Smoking and drinking in relation to oral and pharyn-geal cancer. Cancer Res 1988; 48: 3282 - 7.

4. Depue P. Rising mortality from cancer of the tongue in young adults. $\mathrm{N}$ Engl J Med 1986; 315: 647.

5. Rioboo R. Odontología preventiva y Odontología comunitaria. Tomo II. Cap 40. Estudios epidemiológicos de las enfermedades y alteraciones bucodentales. Ediciones Avances 2002: 1103 - 25.

6. Graham S, Dayal H, Rohrer T, Swanson M, Sultz $H$, Shedd $D$, et al. Dentition, diet, tabacco and alcohol in the epidemiology of oral cancer. J Natl Cancer Inst. 1997;59: 1611 - 8.

7. Blot WJ, Winn DM, Fraumeni JF JR. Oral cancer and mouthwash. J Nalt Cancer Inst. 1983; 79: 25 I - 3.

8. Shiboski CH, Schmidt BL, Jordam RCK. Tongue and Tonsil Carcinoma. Cancer. 2005; 103:9. P.1843 - 49.

9. Dabelsteen E, Roed-Petersen B, Smith CJ, Pindborg JJ. The limitations of exfoliative cytology for the detection of epithelial atypia in oral leukoplakias. Br J Cancer 197I; 25: 21 - 4.

10. Sugerman PB, Savage NW. Exfoliative cytology in clinical oral pathology. Aust Dent J 1996; 4I: 7I - 4.

II. Jones CJ, Pink FE, Sandow PL, Stewart CM, Migliorati CA, Baughman RA. The citobrush cell colector in oral cytology. Oral Surg Oral Med Oral Pathol 1994; 77: 101 - 7.

12. Diniz-Freitas M, García-García A, CrespoAbelleira A, Martins- Carneiro JL, Gándara-Rey JM. Aplicaciones de la citología exfoliativa en el diagnóstico del cáncer oral. Med Oral2004; 9: 355 - 61 .

13. Nagpala JK, Dasa BR. Oral cancer: reviewing the present understanding of its molecular mechanism and exploring the future directions for its directive management. Review. Oral Oncology 2003; 39: $213-21$.

14. Lanfranchi HE, Klein-Szato AJP. Patología de la Mucosa Oral. En: Cabrini R, eds. Anatomía Patológica Bucal. Buenos Aires: Mundi Editores; 1988. P. 301 - 50.

15. Ceccotti EJ, eds. Cínica Estomatológica. SIDA, Cáncer y otras afecciones Buenos Aires: 
Panamericana Editores; 1993. p. I-500.

16. Sawaf MH, Ouhayoun JP, Forest N. Cytokeratin profiles in oral epithelia: review and a new classification. J Biol Buccales 1991; 19: 187 - 98.

17. Cantoa MT, Devesa SS. Oral cavity and pharynx cancer incidence rates in the United States, 1975 1998. Oral Oncology 2002; 38: 610 - 7.

18. Brunotto $M$, Zárate $A M$, Cismondi $A$, Fernández MC, Noher de Halac RI. Valuation of exfoliative cytology as prediction factor in oral mucosa lesions. Med Oral Patol Oral Cir Bucal 2005; I0: Suppl 2: E92 - 102.

19. Silverman S J, Bilimoria KF, Bhargava K, Mani $\mathrm{NJ}$, Shah R. Cytologycal, histological and clinical correlation of precancerous oral lesions in 57,518 industrial workers of Gujarat, India. Acta Cytologica 1977; 21 : 196 - 8.

20. Sciubba JJ. Improving detection of precancerosu and cancerous oral lesions: computer-assisted analysis of the oral brush biopsy. J Am Dent Assoc 1999; I30: I445 - 57.

21. Acha A, Ruesga MT, Rodríguez MJ, Martínez de Pancorbo MA, Aguirre JM. Applications of the oral scraped (exfoliative) cytology in oral cancer and precancer. Med Oral Patol Oral CirBucal 2005; 10: 95 - 102.

22. Warnakulasuriya $S$. Global trends in the epidemiology of oral cancer; the current position. Oral Oncol 2005; I: 27. Abstract K-I.

23. Gallegos HJF. Head and neck cancer. Prognostic factors and prevention.Cir Ciruj 2006; 74: 287 $-293$.

24. De Vita VT, Hellman S, Rosenberg SA. Cancer. Principles and practice of oncology. 5.ed. Philadelphia: Lippincott-Raven; 1997: 92- II3, 228- 37,574 - 622.

25. Santana JC. Atlas de patología del complejo bucal. La Habana: Edit. Científico-Técnica; 1985: $45-63$.

26. Oscar García- Roco Pérez, I Dr. Miguel Arredondo López2 y Dra. María del C. Álvarez Navarro3 Citología exfoliativa en el diagnóstico precoz de lesiones oncológicas bucales Rev Cubana Estomatol 2002; 39 (2).

27. Epstein JB, Zhang L, Rosin M. Advances in the diagnosis of oral premalignant and malignant lesions. J Can Dent Assoc 2002; 68: 617 - 21. 